\title{
Identification of suitable endogenous control genes for microRNA expression profiling of childhood medulloblastoma and human neural stem cells
}

Laura A Genovesi ${ }^{1}$, Denise Anderson ${ }^{2}$, Kim W Carter ${ }^{2}$, Keith M Giles ${ }^{3}$ and Peter B Dallas ${ }^{\text {* }}$

\begin{abstract}
Background: Medulloblastoma (MB) is the most common type of malignant childhood brain tumour. Although deregulated microRNA (miRNA) expression has been linked to MB pathogenesis, the selection of appropriate candidate endogenous control (EC) reference genes for MB miRNA expression profiling studies has not been systematically addressed. In this study we utilised reverse transcriptase quantitative PCR (RT-qPCR) to identify the most appropriate EC reference genes for the accurate normalisation of miRNA expression data in primary human MB specimens and neural stem cells.

Results: Expression profiling of 662 miRNAs and six small nuclear/ nucleolar RNAs in primary human MB specimens, two CD133+ neural stem cell (NSC) populations and two CD133- neural progenitor cell (NPC) populations was performed using TaqMan low-density array (TLDA) cards. Minimal intra-card variability for candidate EC reference gene replicates was observed, however significant inter-card variability was identified between replicates present on both TLDA cards A and B. A panel of 18 potentially suitable EC reference genes was identified for the normalisation of miRNA expression on TLDA cards. These candidates were not significantly differentially expressed between CD133+ NSCs/ CD133- NPCs and primary MB specimens. Of the six sn/snoRNA EC reference genes recommended by the manufacturer, only RNU44 was uniformly expressed between primary MB specimens and CD133+ NSC/ CD133- NPC populations ( $P=0.709 ; F C=1.02$ ). The suitability of candidate $E C$ reference genes was assessed using geNorm and NormFinder software, with hsa-miR-301a and hsa-miR-339-5p found to be the most uniformly expressed EC reference genes on TLDA card A and hsa-miR-425* and RNU24 for TLDA card B.

Conclusions: A panel of 18 potential EC reference genes that were not significantly differentially expressed between CD133+ NSCs/ CD133- NPCs and primary human MB specimens was identified. The top ranked EC reference genes described here should be validated in a larger cohort of specimens to verify their utility as controls for the normalisation of RT-qPCR data generated in MB miRNA expression studies. Importantly, inter-card variability observed between replicates of certain candidate EC reference genes has major implications for the accurate normalisation of miRNA expression data obtained using the miRNA TLDA platform.
\end{abstract}

Keywords: MicroRNA, Medulloblastoma, Neural stem cells, Gene expression profiling, Quantitative RT-PCR

\footnotetext{
* Correspondence: peterd@ichr.uwa.edu.au

'Brain Tumour Research Program, Telethon Institute for Child Health Research, Centre for Child Health Research, University of Western Australia, Perth, Western, Australia

Full list of author information is available at the end of the article
} 


\section{Background}

Medulloblastoma (MB) is the most common malignant paediatric brain tumour, and a major cause of childhood cancer related morbidity and mortality [1]. Several molecular subtypes of MB have been identified on the basis of specific gene expression signatures [2-6], suggesting that different sub-groups may arise from distinct cells of origin. In human MB, a subpopulation of CD133expressing cells was identified that displayed similar properties to NSCs, including self-renewal and multipotency $[7,8]$. Additional studies demonstrated that these putative brain tumour stem cells (BTSCs) were capable of initiating tumour formation in immunodeficient mice [9]. These human data combined with evidence from two different murine MB models [10,11] strongly implicate CD133+ NSCs as a cell of origin for a subset of MB.

MicroRNAs (miRNAs) are a class of short, non-coding RNAs that down-regulate gene expression at a posttranscriptional level $[12,13]$. Functioning as guide molecules for silencing complexes, miRNAs utilise anti-sense complementarity to inhibit the expression of specific messenger RNA (mRNA) targets by either repressing translation and/or inducing deadenylation and subsequent mRNA degradation [13-16]. miRNAs play key regulatory roles in various cellular processes including cell proliferation [17], apoptosis [18] and differentiation [19]. Numerous studies have demonstrated significantly altered miRNA expression patterns in a wide range of human cancer types compared to normal tissues, suggesting that specific miRNAs might act as tumour suppressor genes or oncogenes (Reviewed in [20] and [21]). In addition to their potential as novel molecules for cancer therapy [22], miRNAs represent an emerging class of diagnostic and prognostic markers [23-26].

Adaptations of existing technologies for gene expression profiling including RT-qPCR, chip-based microarrays, and next generation sequencing have enabled the highthroughput profiling of miRNA expression [23,27-31]. The accuracy of these methods is dependent upon correcting for non-biological sample-to-sample variation that could be introduced during the steps from sample preparation to amplification [23]. For miRNA RT-qPCR expression data, several methods have been described to correct for this variation, the most frequent of which is the normalisation to endogenous control (EC) reference genes [32]. An ideal reference gene should be highly expressed, exhibit minimal expression level variation in cells or tissues under investigation, and be of similar length to that of the target gene [33]. Additionally, extraction and quantification efficiency and storage stability of an ideal reference gene should be equivalent to the gene under investigation [33]. However, previous studies have demonstrated that a single universal EC reference gene with these properties for all cell or tissue types is unlikely to exist [34-37]. Normalisation of RT-qPCR data to unreliable reference genes may lead to incorrect quantification of miRNAs of interest [33,38], and the importance of validating suitable candidate EC reference genes in a cell and/or tissue-specific context has been demonstrated previously [33]. Although several research groups have performed miRNA expression profiling studies in primary MB specimens, the validation strategies for the selection of EC genes for normalisation of miRNA gene expression data were not reported [39-45]. Additionally, none of these studies profiled CD133+ NSCs and/or CD133NPCs, instead normalising miRNA expression to EC gene expression levels in human adult and fetal cerebellum. This study describes a robust strategy for the identification of suitable EC genes for the normalisation of miRNA RTqPCR data in MB profiling studies. Using the main selection criteria of good measurability and uniform expression, candidate EC reference genes for miRNA data normalisation were investigated in nine primary $\mathrm{MB}$ specimens, and two populations of CD133+ NSCs and CD133- NPCs. The uniformity of expression of these candidate EC genes was subsequently investigated across all samples, with the relative quantities of miRNAs, hsa-miR-144*, hsa-miR-21* and hsa-miR-923 assessed using five different normalisers, to determine the impact of EC reference gene selection on relative expression of individual miRNAs of interest.

\section{Results}

Inter-card variation for candidate small nuclear (sn) and small nucleolar (snoRNA) EC reference genes on the TLDA platform

miRNA profiling using the TLDA system consists of two cards, A and B. Card A contains three proposed EC references genes, MammU6, RNU44 and RNU48, and card B contains six, MammU6, RNU44, RNU48, RNU24, RNU43 and RNU6B. To evaluate whether there was inter-plate variation between the sn/snoRNA candidate EC reference genes on TLDA card A and card B, Cq values for technical replicates of three candidate EC reference genes, MammU6, RNU48 and RNU44, present on separate TLDA cards were plotted and one way analysis of variance (ANOVA) was applied to assess interplate variation between the technical replicates (Figure 1). Significant variation was observed between the technical replicates of MammU6 $(P=0.025)$ and RNU44 $(P=0.0030)$ on TLDA cards A and B (Figure 2). In contrast, $\mathrm{Cq}$ values for the technical replicates of RNU48 remained relatively stable $(P=0.97)$. The observed differences between EC reference gene replicate $\mathrm{Cq}$ values on TLDA cards A and B suggests that the normalisation of miRNAs is best performed to EC reference genes on the same TLDA card. Based on these results, we assessed 


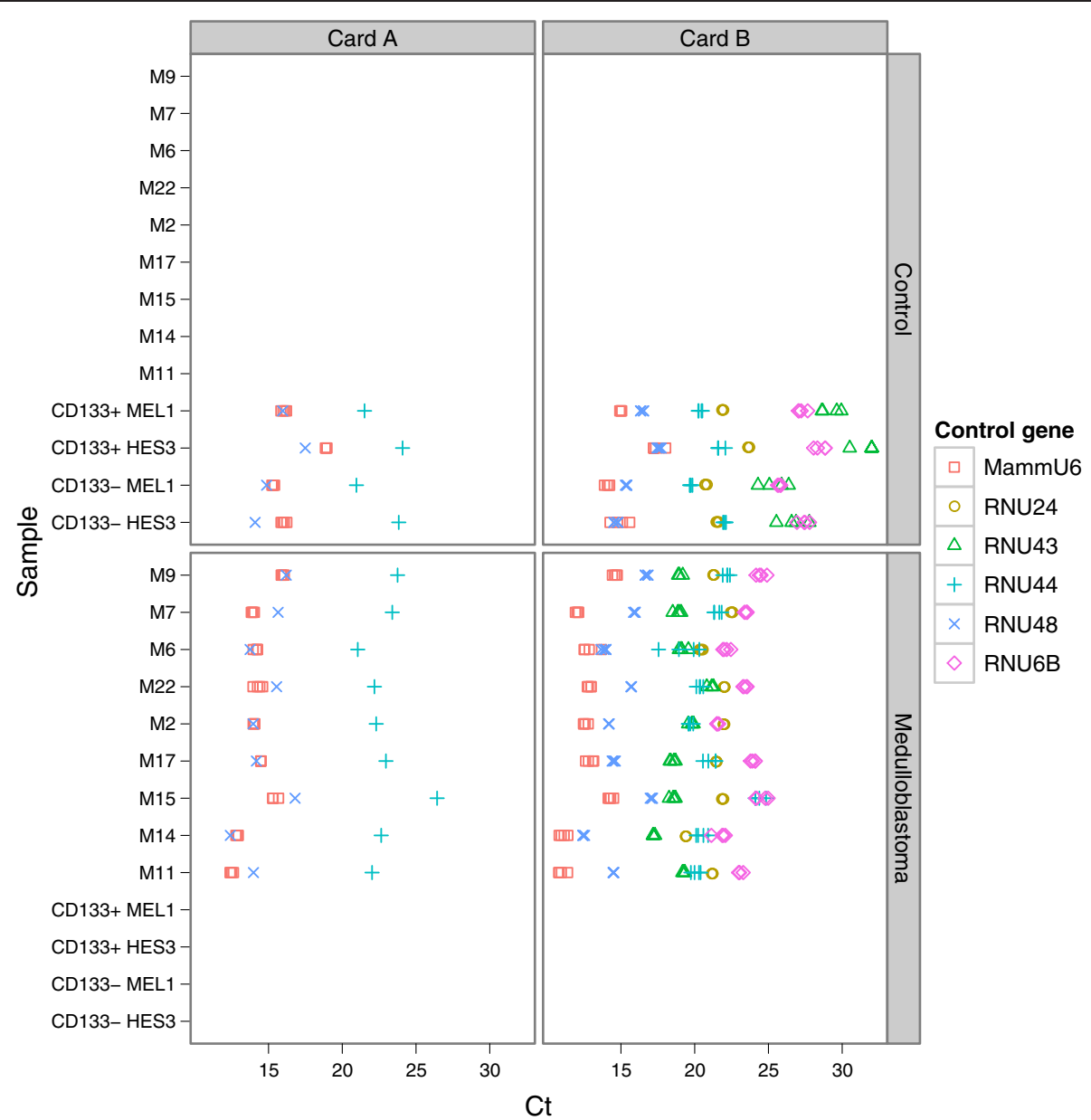

Figure 1 Quantification cycle $(\mathrm{Cq})$ values for the technical replicates of proposed EC reference genes of the (TLDA) cards A (left) and B (right).

the suitability of EC reference genes separately for TLDA cards A and B. As minimal variation was observed for technical replicates of candidate EC reference genes on the same TLDA card, an average of the technical replicates from each TLDA card was employed for further analysis. Because this approach limited the maximum number of proposed sn/snoRNA candidate EC reference genes for each TLDA card, particularly for TLDA card A, we expanded the panel of potential candidate EC reference genes under investigation to include miRNAs that fulfil the appropriate selection criteria outlined in methods.

\section{Expression of candidate EC reference genes in primary MB specimens, CD133+ NSCs and CD133- NPCs}

Suitable candidate EC reference genes must display consistent expression between normal and treatment and/or disease groups. Two sample $t$-tests were employed to assess whether candidate EC reference genes were differentially expressed between primary $\mathrm{MB}$ specimens and
CD133+ NSC/ CD133- NPC populations. For TLDA card A, 12 genes were identified as potentially suitable EC reference genes (Table 1). Of the proposed sn/snoRNA candidate EC reference genes, only RNU44 was uniformly expressed across primary $\mathrm{MB}$ specimens and $\mathrm{CD} 133+$ NSC/CD133- NPC populations $(P=0.71$; fold change $(\mathrm{FC})=1.02) \quad($ Table 1$)$. In contrast, MammU6 was overexpressed in primary $\mathrm{MB}$ specimens compared to NSC/ NPCs $(P=0.049 ; \mathrm{FC}=3.66)$, and while the over-expression of RNU48 did not reach statistical significance $(P=0.35)$, the degree of over-expression observed in primary $\mathrm{MB}$ specimens relative to NSCs/NPCs $(\mathrm{FC}=2.35)$ suggested RNU48 was not an ideal EC reference in this context. In addition to RNU44, a number of candidate miRNAs were uniformly expressed in NSCs and NPCs and primary MB specimens $(P \geq 0.05 ; \mathrm{FC} \leq \pm 1.5)$, highlighting their potential as EC reference genes in this study.

Expression analysis identified six candidate EC reference genes of TLDA card B suitable for data normalisation (Table 1). Of the manufacturer-recommended 


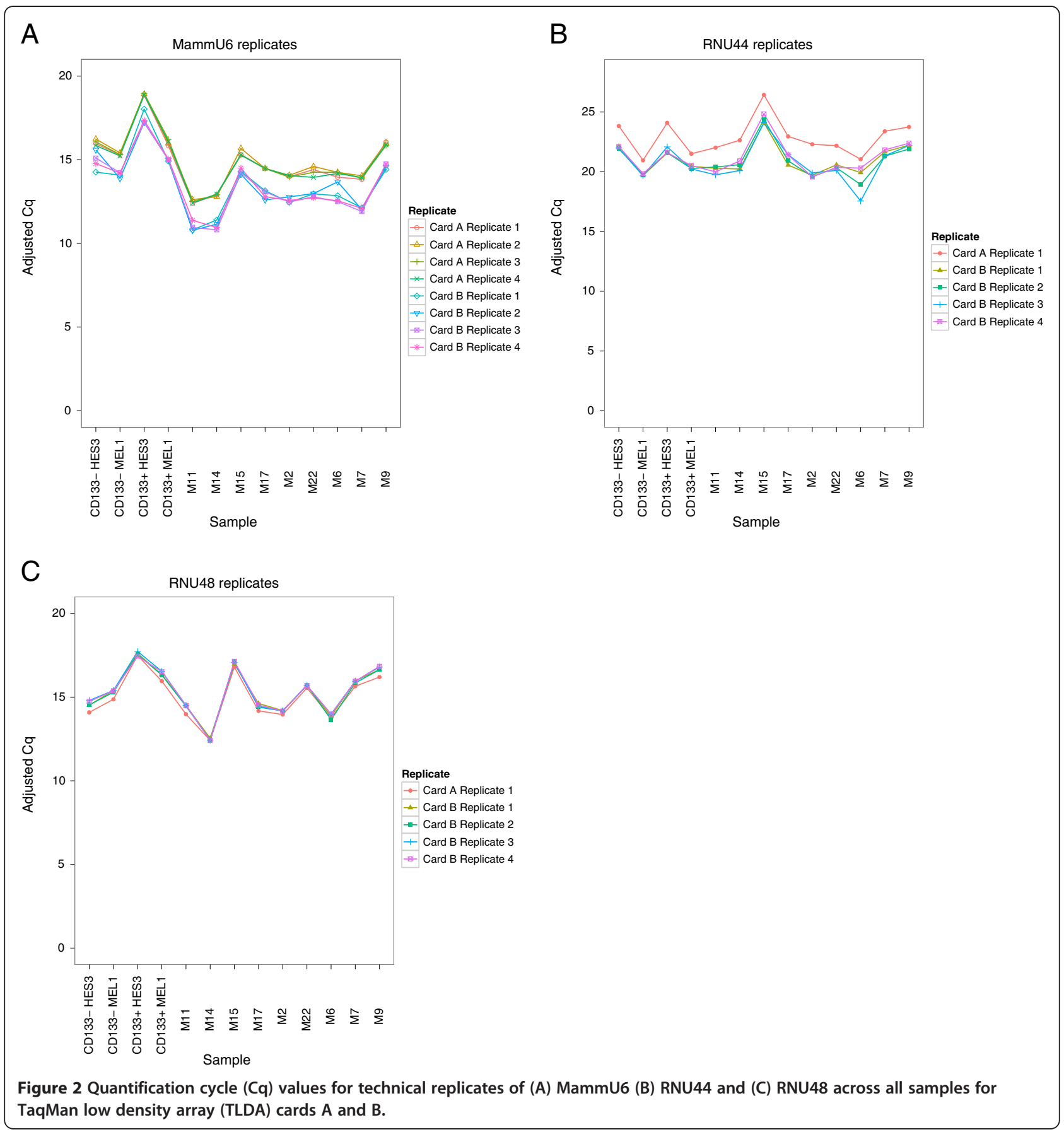

candidate EC reference genes included on TLDA card B, no significant difference was observed for RNU44 $(P=0.97 ; \mathrm{FC}=1.49)$ and RNU24 $(P=0.42 ; \mathrm{FC}=1.19)$ between primary MB samples and NSCs/NPCs. Similar to findings obtained for TLDA card A, increased expression of RNU48 in primary MB specimens $(\mathrm{FC}=2.64)$, although not statistically significant $(P=0.25)$, suggests it may not be the most appropriate EC reference for normalisation. Significantly increased expression of MammU6, RNU6B and RNU43 was identified in primary MB specimens relative to CD133+ NSCs/ CD133- NPCs, particularly for RNU43 $(\sim 500$ fold greater expression in primary $\mathrm{MB}$ ). In addition to the two snoRNAs, RNU44 and RNU24, four miRNAs were also uniformly expressed in the two groups, namely miR-425* $(P=0.49 ; \mathrm{FC}=1.42)$, miR-877 $(P=0.35 ; \mathrm{FC}=$ 1.39), miR-130b* $(P=0.91, \mathrm{FC}=-1.13)$ and miR-181a-2* $(P=0.97 ; \mathrm{FC}=1.48)$.

To compare the abundance of candidate EC reference genes selected for further analysis and to obtain an 
Table 1 Summary of candidate EC reference genes identified for the normalisation of miRNA expression data in MB

\begin{tabular}{|c|c|c|c|c|c|}
\hline Gene & Chromosomal location & $\mathrm{p}$ value & Fold change & Coefficient of variation & Candidate EC gene \\
\hline \multicolumn{6}{|l|}{ Card A } \\
\hline RNU48 & $6 p 21.33$ & 0.351 & 2.36 & 9.52 & No \\
\hline RNU44 & $1 \mathrm{q} 25.1$ & 0.709 & 1.02 & 6.50 & Yes \\
\hline MammU6 & $15 q 23$ & 0.049 & 3.66 & 11.10 & No \\
\hline hsa-miR-133a & $18 q 11.2 / 20 q 13.33$ & 0.797 & 1.16 & 7.05 & Yes \\
\hline hsa-miR-339-5p & $7 p 22.3$ & 0.218 & 1.47 & 5.89 & Yes \\
\hline hsa-miR-214 & $1 \mathrm{q} 24.3$ & 0.853 & 1.36 & 8.45 & Yes \\
\hline hsa-miR-197 & $1 \mathrm{p} 13.3$ & 0.515 & -1.41 & 5.65 & Yes \\
\hline hsa-miR-18a & $13 q 31.3$ & 0.687 & 1.09 & 9.64 & Yes \\
\hline hsa-miR-210 & $11 \mathrm{p} 15.5$ & 0.766 & 1.41 & 6.07 & Yes \\
\hline hsa-miR-328 & $16 q 22.1$ & 0.564 & 1.46 & 7.40 & Yes \\
\hline hsa-miR-301a & $17 q 22$ & 0.384 & 1.32 & 7.23 & Yes \\
\hline hsa-miR-218 & $4 p 15.31$ & 0.592 & 1.14 & 9.86 & Yes \\
\hline hsa-miR-149 & $2 q 37.3$ & 0.647 & -1.36 & 7.97 & Yes \\
\hline hsa-miR-20b & $X q 26.2$ & 0.397 & -1.03 & 9.32 & Yes \\
\hline \multicolumn{6}{|l|}{ Card B } \\
\hline RNU48 & $6 p 21.33$ & 0.250 & 2.64 & 9.50 & $\mathrm{No}$ \\
\hline RNU44 & $1 \mathrm{q} 25.1$ & 0.968 & 1.49 & 6.61 & Yes \\
\hline MammU6 & $15 q 23$ & 0.023 & 4.29 & 13.25 & No \\
\hline RNU24 & $9 q 34$ & 0.423 & 1.19 & 4.77 & Yes \\
\hline RNU43 & $22 q 13$ & $<0.01$ & 498.00 & 21.50 & No \\
\hline RNU6B & 10p13 & $<0.01$ & 14.98 & 9.16 & No \\
\hline hsa-miR-425* & $3 q 21.31$ & 0.493 & 1.42 & 4.53 & Yes \\
\hline hsa-miR-877 & $6 p 21.33$ & 0.350 & 1.39 & 3.33 & Yes \\
\hline hsa-miR-130b* & $22 q 11.2$ & 0.910 & -1.13 & 6.39 & Yes \\
\hline hsa-miR-181a-2* & $9 q 33.3$ & 0.972 & 1.48 & 6.10 & Yes \\
\hline
\end{tabular}

Uniformly and differentially expressed snoRNA candidate EC reference genes from both TLDA cards A and B are listed with chromosomal location.

estimate of their uniformity of expression across CD133 $+\mathrm{NSC} / \mathrm{CD} 133-\mathrm{NPCs}$ and primary MB specimens, Cq values of each candidate $\mathrm{EC}$ reference gene were plotted and the coefficient of variation (CV) for each candidate EC reference gene calculated (Figure 3 ). The mean, CV and range of $\mathrm{Cq}$ values for all candidate $\mathrm{EC}$ reference genes are shown in Table 2. For TLDA card A, miR-20b had the highest expression with a mean Cq of 17.75 , followed by miR-149 and miR-218 both with a mean Cq value of 18.88 . Moderately abundant EC reference genes with mean $\mathrm{Cq}$ values between 20 and 25, included miR18a, miR-133a, miR-197, miR-210, miR-214, miR-328 and RNU44. All candidates displayed low variability, with the least variation across all samples for miR-197 $(\mathrm{CV}=5.65)$, and the largest for miR-218 (CV =9.86). Candidate EC reference genes from TLDA card B were expressed at lower levels, with RNU44 the most highly expressed gene with a mean $\mathrm{Cq}$ value of 20.98. Closely following RNU44 were miR-181a-2* and RNU24, with mean Cq values of 21.22 and 21.53, respectively. miR$425^{*}$ was the lowest expressed EC reference gene, with a mean $\mathrm{Cq}$ value of 24.87. Candidates again displayed low variability, with miR-877 exhibiting the smallest $\mathrm{Cq}$ variation $(\mathrm{CV}=3.33)$, and RNU44 $(\mathrm{CV}=6.60)$ the largest.

\section{Uniformity of candidate reference gene expression in} primary MB specimens, CD133+ NSCs and CD133- NPCs Candidate EC reference genes displaying similar expression levels in CD133+ NSC/ CD133- NPCs and primary $\mathrm{MB}$ specimens were further assessed for uniformity of expression across all samples using geNorm [36] and NormFinder [46] software. RNU48 was also included in this analysis, as although differential expression of this snoRNA, as measured by FC, was observed between primary MB specimens and CD133+ NSCs/ CD133- NPCs, this did not reach statistical significance. The chromosomal location of candidate EC reference genes was assessed to rule out any co-regulation of clustered miRNA 


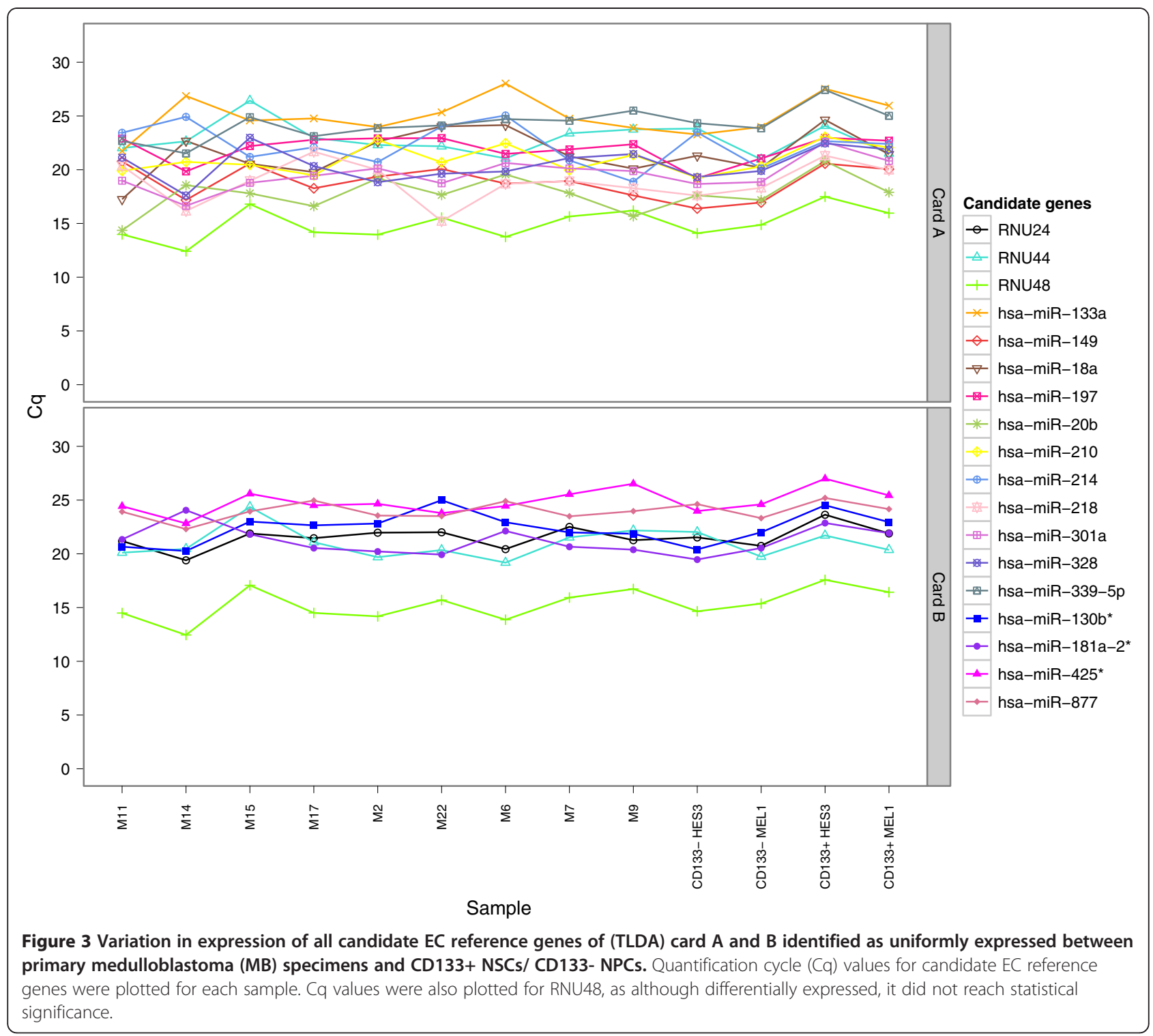

genes transcribed as a single, polycistronic transcript (Table 1), as this would lead to an erroneous choice of an optimum EC gene reference pair using geNorm and NormFinder software. The ranking of the candidate EC reference genes determined by these programs is summarised in Table 3, and consistent results were obtained for both TLDA card A and card B. Normfinder and geNorm identified hsa-miR-301a and hsa-miR-339-5p as the two most uniformly expressed EC reference genes on TLDA card A, closely followed by hsa-miR-210 and RNU48. Normfinder identified the geometric mean of hsa-miR-339-5p and hsa-miR-301a as the most stable pair of EC reference genes, with a lower combined stability value of $M=0.116$ when compared to the stability values of the EC reference genes alone (Table 3). This suggests that the combination of hsa-miR-339-5p and hsa-miR- 301a should be used for data normalisation in preference to the single EC reference genes.

Of the candidate EC reference genes on TLDA card B, hsa-miR-425* and RNU24 were the two most uniformly expressed EC reference genes, followed by RNU48 and hsa-miR-877 (Table 3). The combination of hsa-miR-425* and RNU24 displayed a much lower stability value $(M=0.078)$ than either of the candidate EC reference genes alone, indicating that the geometric mean of these EC reference genes is the most suitable for miRNA expression normalisation of TLDA card B. Although both programs listed RNU48 in the top four most uniformly expressed candidate EC reference genes of TLDA card A and card B, it was not considered ideal due to its differential expression between primary $M B$ specimens and CD133+ NSCs/CD133- NPCs. 
Table 2 Summary statistics of Cq values for candidate EC reference genes

\begin{tabular}{|c|c|c|c|c|}
\hline Gene & Mean & Minimum & Maximum & CV \\
\hline \multicolumn{5}{|l|}{ Card A } \\
\hline RNU48 & 14.99 & 12.41 & 17.49 & 9.52 \\
\hline RNU44 & 22.85 & 20.96 & 26.43 & 6.50 \\
\hline hsa-miR-133a & 24.97 & 21.74 & 28.02 & 7.05 \\
\hline hsa-miR-339-5p & 24.28 & 21.52 & 27.43 & 5.89 \\
\hline hsa-miR-214 & 22.29 & 18.88 & 25.05 & 8.45 \\
\hline hsa-miR-197 & 21.94 & 19.20 & 22.97 & 5.65 \\
\hline hsa-miR-18a & 21.54 & 17.25 & 24.64 & 9.64 \\
\hline hsa-miR-210 & 20.96 & 19.25 & 23.06 & 6.07 \\
\hline hsa-miR-328 & 20.50 & 17.61 & 22.98 & 7.40 \\
\hline hsa-miR-301a & 19.56 & 16.64 & 22.58 & 7.23 \\
\hline hsa-miR-218 & 18.88 & 15.21 & 21.68 & 9.86 \\
\hline hsa-miR-149 & 18.88 & 16.39 & 20.77 & 7.97 \\
\hline hsa-miR-20b & 17.75 & 14.36 & 20.81 & 9.32 \\
\hline \multicolumn{5}{|l|}{ Card B } \\
\hline RNU48 & 15.30 & 12.45 & 17.58 & 9.50 \\
\hline RNU44 & 20.98 & 19.18 & 24.36 & 6.60 \\
\hline RNU24 & 21.53 & 19.40 & 23.64 & 4.77 \\
\hline hsa-miR-425* & 24.87 & 22.83 & 26.99 & 4.53 \\
\hline hsa-miR-877 & 23.99 & 22.30 & 25.20 & 3.33 \\
\hline hsa-miR-130b* & 22.39 & 20.26 & 24.99 & 6.39 \\
\hline hsa-miR-181a-2* & 21.22 & 19.47 & 24.06 & 6.10 \\
\hline
\end{tabular}

Impact of EC reference genes on the relative quantification of individual miRNAs

To demonstrate the impact of selected EC reference genes on the results, we measured the expression of miRNAs relative to several candidate EC reference genes identified in this investigation. Specific candidate snoRNA EC reference genes on TLDA card B were highly differentially expressed in primary $\mathrm{MB}$ specimens relative to CD133+ NSCs and CD133- NPCs, and therefore, as proof of concept, the normalisation of individual miRNAs focused upon TLDA card B miRNAs and candidate $\mathrm{EC}$ reference genes. In primary $\mathrm{MB}$ specimens, the over-expression of three TLDA card B miRNAs, hsamiR-144*, hsa-miR-21* and hsa-miR-923, was previously described [47]. For this analysis, normalisation of these three miRNAs was performed using top ranked candidate EC reference genes identified by geNorm and Normfinder, including hsa-miR-425*, RNU24, hsa-miR877, and the EC reference pair, RNU24/ hsa-miR-425\%. In addition, target miRNA expression was also established relative to the significantly over-expressed ( $\sim 500$ fold) candidate EC reference gene, RNU43. Normalisation using uniformly expressed candidate EC reference genes did not influence the directionality of differential
Table 3 Candidate EC reference genes ranked according to their expression stability as calculated by the geNorm and NormFinder algorithms

\begin{tabular}{|c|c|c|c|c|}
\hline \multirow[t]{2}{*}{ Rank } & \multicolumn{2}{|c|}{ Normfinder } & \multicolumn{2}{|c|}{ geNorm } \\
\hline & Gene & Stability & Gene & Stability (M) \\
\hline \multicolumn{5}{|l|}{ Card A } \\
\hline 1 & hsa-miR-339-5p & 0.16 & hsa-miR-301a & 1.589 \\
\hline 2 & hsa-miR-301a & 0.16 & hsa-miR-339-5p & 1.603 \\
\hline 3 & RNU48 & 0.2 & hsa-miR-210 & 1.634 \\
\hline 4 & hsa-miR-210 & 0.22 & RNU48 & 1.680 \\
\hline 5 & hsa-miR-328 & 0.26 & hsa-miR-197 & 1.726 \\
\hline 6 & hsa-miR-20b & 0.28 & hsa-miR-328 & 1.785 \\
\hline 7 & hsa-miR-197 & 0.3 & hsa-miR-149 & 1.794 \\
\hline 8 & hsa-miR-133a & 0.3 & hsa-miR-20b & 1.841 \\
\hline 9 & hsa-miR-149 & 0.31 & hsa-miR-133a & 1.954 \\
\hline 10 & hsa-miR-218 & 0.32 & RNU44 & 2.074 \\
\hline 11 & hsa-miR-18a & 0.390 & hsa-miR-18a & 2.169 \\
\hline 12 & RNU44 & 0.49 & hsa-miR-218 & 2.188 \\
\hline 13 & hsa-miR-214 & 0.59 & hsa-miR-214 & 2.505 \\
\hline \multicolumn{5}{|l|}{ Card B } \\
\hline 1 & hsa-miR-425* & 0.11 & hsa-miR-425* & 1.177 \\
\hline 2 & RNU24 & 0.11 & RNU24 & 1.180 \\
\hline 3 & RNU48 & 0.16 & $\mathrm{RNU} 48$ & 1.299 \\
\hline 4 & hsa-miR-877 & 0.23 & hsa-miR-877 & 1.301 \\
\hline 5 & hsa-miR-130b & 0.32 & hsa-miR-130b & 1.538 \\
\hline 6 & hsa-miR-181a-2* & 0.34 & RNU44 & 1.569 \\
\hline 7 & RNU44 & 0.39 & hsa-miR-181a-2* & 1.860 \\
\hline
\end{tabular}

expression of miRNAs, with consistent over-expression of each target miRNA observed in primary MB specimens relative to CD133 + NSCs/CD133- NPCs (Figure 4). In contrast, down-regulation of all three miRNAs was observed when RNU43 was utilised for data normalisation, due to the significant over-expression of RNU43 in primary $\mathrm{MB}$ specimens relative to target miRNA expression. Taken together, these results emphasise that establishing target miRNA expression levels for a particular tissue and/or disease state is strongly influenced by the candidate EC reference gene chosen for data normalisation.

\section{Discussion}

The importance of validating suitable candidate EC reference genes in a cell and/or tissue-specific context is well documented, and a single universal reference gene for all tissue types is unlikely to exist [33-37]. Indeed, in the context of cancer, a disease in which the cell of origin is unknown or inadequately characterised in many cases, the selection of appropriate EC reference genes is particularly difficult. Although the cells of origin of the 


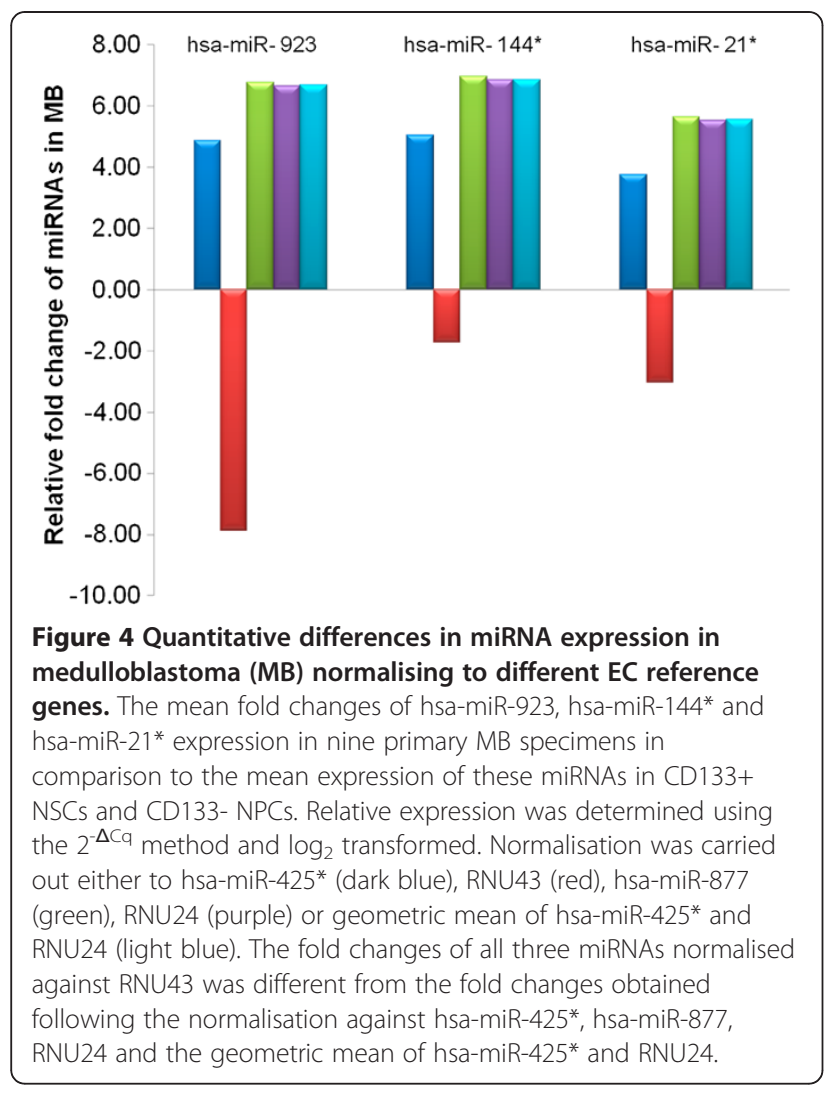

at least four molecular sub-types of human MB have not been conclusively identified, the available murine data suggest that $\mathrm{SHH}$-dependent $\mathrm{MB}$ may have multiple cells of origin including cerebellar granule precursor cells, NSCs [48,49], and cochlear nuclei of the lower rhombic lip [50]. Other mouse models suggest that Group 3 MB arise from CD133+ NSCs [11], and WNTdriven tumours originate from progenitor cells of the dorsal brainstem [51]. The cell of origin of Group 4 tumors has not yet been determined. On this basis, the normalisation of gene expression levels in primary $M B$ specimens to levels in whole foetal or adult cerebellum, which represent heterogeneous tissues at different developmental stages, although generally accepted, is likely to be sub-optimal. Similarly, the normalisation of gene expression levels in primary human $\mathrm{MB}$ to those in ESC-derived NSCs as described here, may be more appropriate for the analysis of specific MB subtypes (e.g., SHH or Group $3 \mathrm{MB}$ ), or stem cell regulatory pathways in MB pathogenesis. The assessment of miRNA expression levels in a larger cohort of primary MB specimens relative to NSCs, and an improved understanding of MB cells of origin will be necessary to address these issues. In the meantime, the data reported here represent an important complementary resource for future $\mathrm{MB}$ miRNA profiling studies.
Normalisation based on predefined invariant EC genes such as sn/snoRNAs is a commonly utilised approach in miRNA RT-qPCR profiling analysis [52]. However, in the context of this study, the majority of pre-defined small nuclear and small nucleolar RNAs were not suitable as EC reference genes for miRNA data normalisation. Small non-coding RNAs other than miRNAs do not mirror the physiochemical properties of miRNA molecules, suggesting that normalisation of miRNA expression data should be performed with reference genes belonging to the same RNA class $[33,36]$. The selection of invariant miRNAs identified by algorithms specifically tailored to reference gene ranking by stepwise elimination of the least stable gene such as geNorm [36], or through statistical linear mixed-effects modelling such as Normfinder [46] have been previously identified as superior to sn/snoRNAbased normalisation [33,53]. Indeed, we identified various miRNAs that were more uniformly expressed across all samples and therefore represent more suitable EC reference genes than the sn/snoRNAs proposed by the manufacturer, a finding that was consistent with a number of previous studies [38,54-57]. Thus, our data provide further evidence to suggest that miRNAs, in comparison to other classes of small non-coding RNAs, may be more suitable EC reference genes for the normalisation of miRNA expression data, providing their expression meets the general consensus of moderate abundance and consistency across all samples.

The overall uniformity of expression is a major determinant for an ideal EC reference gene [33]. Our initial statistical analyses identified a number of candidate EC reference genes uniformly expressed across experimental groups, with both NormFinder and geNorm identifying hsa-miR-339-5p and hsa-miR-301a as the most stable EC reference genes for TLDA card A, and hsa-miR-425* and RNU24 as the most stable for TLDA card B. The practical consequences of miRNA normalisation were then evaluated using TLDA card B candidate EC reference genes and miRNAs as a case study. As evident from our results, inappropriate use of EC reference genes can significantly impact upon target miRNA quantitation. With the use of suitable EC reference genes, including hsa-miR-425*, RNU24, hsa-miR-877 and EC reference pair, RNU24/hsa-miR-425*, over-expression of all three miRNAs (hsa-miR-21*, hsa-miR-144* and hsamiR-923) was identified, as previously reported [47]. However, when an inappropriate EC reference gene, RNU43, was used for expression data normalisation, the down-regulation of all three miRNAs was observed. Several previously published studies in a range of other tissue types have reported similar misleading results when inappropriate EC reference genes were used $[33,38,54,55,58]$, highlighting the importance of selecting appropriate and validated EC reference genes for miRNA 
expression data normalisation. Although several previous MB miRNA profiling studies utilised RNU6B and RNU66 as an EC reference gene pair for normalisation, the validation of these reference genes was not reported $[39,41,45]$. It is important to note that these previous studies normalised miRNA expression to EC reference levels in human normal adult and foetal cerebellum, rather than CD133+ NSCs/CD133- NPCs profiled in this investigation. The findings obtained in this study indicated that RNU6B was significantly differentially expressed in primary $\mathrm{MB}$ specimens relative to $\mathrm{CD} 133+$ NSCs/CD133- NPCs, and therefore was not a suitable EC reference gene for normalisation. Unfortunately, the suitability of RNU66 could not be assessed in this investigation, as the RNU66 miRNA assay was not included in the V2.0 TLDA cards. An additional MB miRNA profiling study utilised RNU48 as a single reference gene for normalisation of miRNA expression data [44]. Differential expression of RNU48 was observed in this study, however this did not reach statistical significance and again, a direct comparison was not possible due to the different normal control tissues being profiled. Combined, these findings reiterate the importance of validating suitable candidate EC reference genes in the relevant cell and/or tissue under investigation.

The performance of miRNA profiling by the highthroughput TLDA miRNA expression profiling system has been evaluated in several studies, with high reproducibility observed for technical replicates of miRNA assays located on the same TLDA card [53,59-61]. While we also observed minimal intra-card variability for all candidate EC reference genes, significant inter-card variability was apparent between replicates of MammU6 and RNU44 on TLDA cards A and B. Similar findings were obtained in a previous study, where differential expression of MammU6 and RNU44, but not RNU48, was observed between the same samples profiled on TLDA cards A and B [61]. For the TLDA system, two separate Megaplex primer pools are required for reverse transcription (RT) of RNA and pre-amplification of cDNA prior to application to cards $\mathrm{A}$ and $\mathrm{B}$. We propose that the inter-card variability for replicates of MammU6 and RNU44 was perhaps a direct consequence of differential RT and pre-amplification efficiencies of these sn/snoRNAs associated with the separate reactions. Previously, the variable expression of a subset of miRNAs was largely attributed to the altered efficiencies of the Megaplex RT and pre-amplification reactions [53]. Whilst a systematic evaluation of the inter-card reproducibility of the miRNA TLDA platform has not been reported, the findings obtained in this study have important implications for the normalisation of miRNA expression data obtained using this system. Future studies should carefully assess the potential for inter-card/plate variability of the associated platform prior to the assessment of candidate EC reference genes for data normalisation.

\section{Conclusion}

A panel of 18 potential EC reference genes that were not significantly differentially expressed between CD133+ NSCs/ CD133- NPCs and primary human MB specimens was identified. Based on our findings, EC reference gene pairs hsa-miR-301a and hsa-miR-339-5p and hsa-miR-425* and RNU24 are recommended for the normalisation of miRNAs on TLDA card A and card B, respectively, in primary $\mathrm{MB}$ specimens relative to ESC-derived NSCs/ NPCs. The top ranked EC reference genes described here should be validated in a larger cohort of specimens to verify their utility as controls for the normalisation of RT-qPCR data generated in MB miRNA expression studies. More broadly, inter-card variability observed between replicates of certain candidate EC reference genes has major implications for the accurate normalisation of miRNA expression data obtained using the miRNA TLDA platform.

\section{Methods}

\section{Patient samples}

Nine MB samples were collected from children treated at Princess Margaret Hospital (PMH) in Perth, Western Australia. Tumour tissue was embedded in optimal cutting temperature compound (OCT) and snap-frozen. The age of patients, gender distribution and molecular sub-type of each primary MB specimen have been previously described (Table 4) [47]. Written approval to undertake this study was obtained from the PMH human ethics committee. Written consent to use tumour material for research purposes was obtained from the parents of patients according to $\mathrm{PMH}$ ethics

Table 4 Clinical data and molecular sub-type of primary MB specimens utilised in this investigation

\begin{tabular}{lllll}
\hline Sample & Gender & Age & M Status & Sub-type \\
\hline M2 & Female & 3 & Unknown & B \\
M6 & Male & 7 & M+ & C \\
M7 & Male & 1.5 & M0 & B \\
M9 & Male & 2 & Unknown & N/A \\
M11 & Male & 5 & M+ & DE \\
M14 & Female & 3 & M0 & C \\
M15 & Male & 3 & M+ & DE \\
M17 & Male & 4 & M+ & DE \\
M22 & Male & 1 & Unknown & N/A \\
\hline
\end{tabular}

Metastatic status was defined as M0 (no metastasis) or M+ (distant metastases). Primary specimens defined as "N/A" were not available for sub-typing analysis [47]. Sub-type designations were based on the data of Kool et al., [3]. 
committee guidelines. All tumour material was deidentified to ensure patient anonymity.

\section{Neurosphere maintenance and flow cytometry}

Human NSCs propagated as neurospheres were derived from human embryonic stem cell (ESC) lines hES3 (WiCell Research Institute, Madison, WI, USA) and MEL1 (StemCore, Melbourne, Australia) using protocols described previously [62,63]. Dissociation of neurospheres and isolation of CD133+ NSCs by flow cytometry was performed as described previously [64]. Enrichment of CD133- NPCs was $100 \%$ for both hES3 and MEL1 ESC lines, with the enrichment of CD133+ NSCs being $81.1 \%$ and $97 \%$ for the hES3 and MEL1 ESC lines, respectively.

\section{Small RNA isolation and enrichment}

RNA enriched for small RNAs was isolated from primary specimens, cell lines and NSC/NPCs using the miRNeasy mini kit (Qiagen, Melbourne Australia), as described previously [47]. RNA quantity and purity was estimated by the ratio of absorbance at $260 \mathrm{~nm}$ to that at $280 \mathrm{~nm}\left(\mathrm{OD}_{260}: \mathrm{OD}_{280}\right)$, with ratios of between 1.8 and 2.0 being considered optimal.

\section{miRNA expression profiling}

miRNA profiling was performed using quantitative realtime RT-PCR (RT-qPCR) utilising pre-printed TaqMan low density array (TLDA) microfluidic cards (Human miR v2.0, Applied Biosystems) as described [47]. Each TLDA card set contained MGB-labelled probes specific to 662 mature miRNAs plus six proposed small nuclear (sn)/ small nucleolar (sno) RNA candidate EC reference genes (MammU6, RNU44, RNU48, RNU24, RNU43, RNU6B). Assays for three of these candidate EC reference genes (MammU6, RNU44 and RNU48) were included on TLDA cards A and B, and the other three (RNU24, RNU43 and RNU6B) were only present on TLDA card B. Details of these candidate $\mathrm{EC}$ reference genes and the number of technical replicates found on TLDA cards A and B are provided in Table 5. Pre-processing of raw TLDA data files consisted of threshold and baseline corrections for each sample, with each amplification plot assessed to confirm that the quantification cycle $(\mathrm{Cq})$ value corresponded with the midpoint of logarithmic amplification (SDS 2.3, Life Technologies, Melbourne). Cq values greater than 32 were imputed to 32 according to the manufacturer's technical recommendation.

\section{Statistical analysis}

Statistics used for the filtering of candidate EC reference genes from TLDA cards were calculated in $\mathrm{R}$ statistical environment version 2.13.0 [65] and candidates were filtered according to three criteria: (1) candidate EC reference genes must be moderately to highly expressed across all samples, defined as a mean $\mathrm{Cq}$ of $\leq 25$; (2) candidate EC reference genes must be consistently expressed between the combined CD133+ NSCs and CD133- NPCs, and primary MB specimens, with significantly differentially expressed miRNAs identified using a two sample $t$-test $(P<0.05)$ and a absolute differential expression fold change (FC) value of 1.5 ( $|\mathrm{FC}| \geq 1.5$ ); (3) candidate EC reference genes must be uniformly expressed across all samples, with a coefficient of variation $(\mathrm{CV})<10$. The coefficient of variation $(\mathrm{CV})$ is the ratio of the standard deviation to the mean expressed as a percentage and is a measure of expression variability of candidate EC reference genes across all samples. Candidate EC reference genes that met these criteria (see Table 2) were deemed suitable for subsequent analysis using the geNorm [36] and NormFinder [46] software. The geNorm algorithm calculates the gene expression stability measure $(\mathrm{M})$ for a candidate EC reference gene based upon the average pairwise variation $(\mathrm{V})$ for that gene against all other tested candidate EC reference genes [36]. NormFinder, a Microsoft Excel add-in, is based upon an analysis of variance (ANOVA) model which estimates an intra- and inter-group variation to provide a stability value for each candidate EC reference gene [46]. Normfinder provides the single most stable reference gene, in addition to an EC reference gene pair that has a stability value less than that of the single EC reference gene. Prior to NormFinder analyses, $\mathrm{Cq}$ values were converted to relative expression values using the $2^{-}$ ${ }^{\mathrm{Cq}}$ method [66]. In both programs, lower values indicate increased stability of EC reference genes, and therefore

Table 5 Small nuclear and small nucleolar RNA candidate EC reference genes included on TLDA cards

\begin{tabular}{lllllll}
\hline Gene & Length $(\mathbf{n t})$ & RNA species & Entrez Gene ID & Replicates on Card A & Replicates on Card B & Total number of replicates \\
\hline RNU48 & 63 & snoRNA & NR_002745 & 1 & 4 & 5 \\
RNU44 & 61 & snoRNA & NR_002750 & 1 & 4 & 5 \\
RNU43 & 62 & snoRNA & NR_002439 & 0 & 4 & 4 \\
RNU24 & 75 & snoRNA & NR_002447 & 0 & 4 & 4 \\
RNU6B & 45 & snoRNA & NR_002752 & 0 & 4 & 4 \\
MammU6 & 106 & snRNA & NR_004394 & 4 & 4 & 8 \\
\hline
\end{tabular}

Accession numbers for each of the candidate EC reference genes and number of replicates per card are also listed. 
allow for the ranking of genes on this basis. Cq values of the target TLDA card B miRNAs hsa-miR-923, hsa-miR-144* and hsa-miR-21* were further normalised to each selected EC reference gene and relative expression was determined using the $2^{-\Delta \mathrm{Cq}}$ method, where $\Delta \mathrm{Cq}=\left(\mathrm{Cq}_{\mathrm{miR}}-\mathrm{Cq}_{\text {endogenous control gene }}\right)$. To identify differential expression of individual miRNAs, the normalised means $\left[\log _{2}\left(2^{-\Delta C q}\right)\right]$ of expression levels for each miRNA in primary $\mathrm{MB}$ specimens were compared to the normalised means $\left(\log _{2}\left(2^{-\Delta C q}\right)\right.$ of expression for that miRNA in CD133+ NSCs and CD133-NPCs. For CD133+ NSCs and CD133- NPCs, normalised means $\left(\log _{2}\left(2^{-\Delta C q}\right)\right.$ of expression levels were obtained by averaging the expression of individual miRNAs from both hES3 and Mel1, therefore representing a pool of two ESC cell lines.

\section{Availability of supporting data}

The data sets supporting the results of this article are available from the Telethon Institute for Child Health Research (TICHR) repository: http://bioinformatics. childhealthresearch.org.au/datasets/.

\section{Competing interests}

The authors have declared that no competing interests exist.

\section{Authors' contributions}

Conceived and designed the experiments: LAG DA KMG PBD, Performed the experiments: LAG. Analysed the data: LAG DA, Contributed reagents/ materials/analysis tools: PBD, KWC, Wrote the paper: LAG DA KWC KMG PBD. All authors read and approved the final manuscript.

\section{Acknowledgements}

This project was generously supported by the Telethon Adventurers. Additional funds were received from a University of Western Australia Raine Priming Grant (PBD), Cancer Council of Western Australia project grant 1006115 (PBD, KWC, KMG), and the Stan and Jean Perron Top-up scholarship (LAG). The authors also gratefully acknowledge The Brady Cancer Support Foundation for the purchase of critical equipment.

\section{Author details}

'Brain Tumour Research Program, Telethon Institute for Child Health Research, Centre for Child Health Research, University of Western Australia, Perth, Western, Australia. ${ }^{2}$ Division of Bioinformatics and Biostatistics, Telethon Institute for Child Health Research, Centre for Child Health Research, University of Western Australia, Perth, Western, Australia. ${ }^{3}$ Laboratory for Cancer Medicine, Western Australian Institute for Medical Research, Centre for Medical Research, University of Western Australia, Perth Western Australia.

Received: 31 May 2012 Accepted: 12 September 2012 Published: 14 September 2012

\section{References}

1. Giangaspero F, et al: Medulloblastoma, in WHO Classification of Tumours of the Central Nervous System. In. Edited by Louis DN. Lyon: International Agency for Research on Cancer (IARC); 2007:132-140.

2. Thompson MC, et al: Genomics identifies medulloblastoma subgroups that are enriched for specific genetic alterations. J Clin Oncol 2006, 24(12):1924-31.

3. Kool M, et al: Integrated genomics identifies five medulloblastoma subtypes with distinct genetic profiles, pathway signatures and clinicopathological features. PLOS One 2008, 3(8):e3088.
4. Northcott PA, et al: Medulloblastoma Comprises Four Distinct Molecular Variants. J Clin Oncol 2010, 29(10):1408-14.

5. Cho YJ, et al: Integrative Genomic Analysis of Medulloblastoma Identifies a Molecular Subgroup That Drives Poor Clinical Outcome. J Clin Oncol 2011, 29(11):1424-30.

6. Kool M, et al: Molecular subgroups of medulloblastoma: an international meta-analysis of transcriptome, genetic aberrations, and clinical data of WNT, SHH, Group 3, and Group 4 medulloblastomas. Acta Neuropathol 2012, 123(4):473-84.

7. Hemmati HD, et al: Cancerous stem cells can arise from pediatric brain tumors. Proc Natl Acad Sci USA 2003, 100(25):15178-83.

8. Singh SK, et al: Cancer stem cells in nervous system tumors. Oncogene 2004, 23(43):7267-73.

9. Singh SK, et al: Identification of human brain tumour initiating cells. Nature 2004, 432(7015):396-401.

10. Sutter $\mathrm{R}$, et al: Cerebellar stem cells act as medulloblastoma-initiating cells in a mouse model and a neural stem cell signature characterizes a subset of human medulloblastomas. Oncogene 2010, 29(12):1845-56.

11. Pei $Y$, et al: An animal model of MYC-driven medulloblastoma. Cancer Cell 2012, 21(2):155-67.

12. Lai EC: microRNAs: runts of the genome assert themselves. Curr Bio 2003, 13(23):925-936.

13. Bartel DP: MicroRNAs: genomics, biogenesis, mechanism, and function. Cell 2004, 116(2):281-97.

14. Eulalio A, Huntzinger $E$, Izaurralde E: Getting to the root of miRNAmediated gene silencing. Cell 2008, 132(1):9-14.

15. Filipowicz W, Bhattacharyya SN, Sonenberg N: Mechanisms of posttranscriptional regulation by microRNAs: are the answers in sight? Nat Rev Genet 2008, 9(2):102-14.

16. Chekulaeva M, Filipowicz W: Mechanisms of miRNA-mediated posttranscriptional regulation in animal cells. Curr Opin Cell Biol 2009, 21(3):452-60.

17. Cheng $A M$, et al: Antisense inhibition of human miRNAs and indications for an involvement of miRNA in cell growth and apoptosis. Nucleic Acids Res 2005, 33(4):1290-7.

18. Xu P, Guo M, Hay BA: MicroRNAs and the regulation of cell death. Trends Genet 2004, 20(12):617-24.

19. Chen $C Z$, et al: MicroRNAs modulate hematopoietic lineage differentiation. Science 2004, 303(5654):83-6.

20. Cowland JB, Hother C, Gronbaek K: MicroRNAs and cancer. APMIS 2007, 115(10):1090-106.

21. Garzon R, Calin GA, Croce CM: MicroRNAs in Cancer. Annu Rev Med 2009, 60:167-79.

22. Nana-Sinkam SP, Croce CM: MicroRNAs as therapeutic targets in cancer. Transl Res 2011, 157(4):216-25.

23. Dahlgaard J, et al: Analytical variables influencing the performance of a miRNA based laboratory assay for prediction of relapse in stage I nonsmall cell lung cancer (NSCLC). BMC Res Notes 2011, 4:424.

24. Gattolliat $\mathrm{CH}$, et al: Expression of miR-487b and miR-410 encoded by $14 q 32.31$ locus is a prognostic marker in neuroblastoma. $\mathrm{Br} \mathrm{J}$ Cancer 2011, 105(9):1352-1361.

25. Wang ZX, et al: Prognostic significance of serum miRNA-21 expression in human non-small cell lung cancer. J Surg Oncol 2011, 104(7):847-51.

26. $\mathrm{Yu} \mathrm{G}$, et al: Prognostic values of the miR-17-92 cluster and its paralogs in colon cancer. J Surg Oncol 2012, 106(3):232-7.

27. Krichevsky AM, et al: A microRNA array reveals extensive regulation of microRNAs during brain development. RNA 2003, 9(10):1274-81.

28. Nelson PT, et al: Microarray-based, high-throughput gene expression profiling of microRNAs. Nat Methods 2004, 1(2):155-61.

29. Thomson JM, et al: A custom microarray platform for analysis of microRNA gene expression. Nat Methods 2004, 1(1):47-53.

30. Chen $C$, et al: Real-time quantification of microRNAs by stem-loop RTPCR. Nucleic Acids Res 2005, 33(20):e179.

31. Hafner $M$, et al: Identification of microRNAs and other small regulatory RNAs using cDNA library sequencing. Methods 2008, 44(1):3-12.

32. Deo A, Carlsson J, Lindlof A: How to choose a normalization strategy for miRNA quantitative real-time (qPCR) arrays. J Bioinform Comput Biol 2011, 9(6):795-812.

33. Peltier HJ, Latham GJ: Normalization of microRNA expression levels in quantitative RT-PCR assays: identification of suitable reference RNA 
targets in normal and cancerous human solid tissues. RNA 2008, 14(5):844-52.

34. Thellin $\mathrm{O}$, et al: Housekeeping genes as internal standards: use and limits. J Biotechnol 1999, 75(2-3):291-5.

35. Bustin SA: Absolute quantification of mRNA using real-time reverse transcription polymerase chain reaction assays. J Mol Endocrinol 2000, 25(2):169-93.

36. Vandesompele J, et al: Accurate normalization of real-time quantitative RT-PCR data by geometric averaging of multiple internal control genes. Genome Biol 2002, 3(7):RESEARCH0034. RESEARCH0034.

37. Haller $F$, et al: Equivalence test in quantitative reverse transcription polymerase chain reaction: confirmation of reference genes suitable for normalization. Anal Biochem 2004, 335(1):RESEARCH0034-9.

38. Davoren PA, et al: Identification of suitable endogenous control genes for microRNA gene expression analysis in human breast cancer. BMC Mol Biol 2008, 9:76.

39. Ferretti $E$, et al: Concerted microRNA control of Hedgehog signalling in cerebellar neuronal progenitor and tumour cells. EMBO J 2008, 27(19):2616-27.

40. Uziel T, et al: The miR-17 92 cluster collaborates with the Sonic Hedgehog pathway in medulloblastoma. Proc Natl Acad Sci USA 2009, 106(8):2812-7.

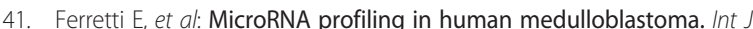
Cancer 2009, 124(3):568-77.

42. Garzia L, et al: MicroRNA-199b-5p impairs cancer stem cells through negative regulation of HES1 in medulloblastoma. PLoS One 2009, 4(3):e4998.

43. Northcott PA, et al: The miR-17/92 polycistron is up-regulated in sonic hedgehog-driven medulloblastomas and induced by $\mathrm{N}$-myc in sonic hedgehog-treated cerebellar neural precursors. Cancer Res 2009, 69(8):3249-55

44. Gokhale A, et al: Distinctive microRNA signature of medulloblastomas associated with the WNT signaling pathway. J Cancer Res Ther 2010, 6(4):521-9.

45. Venkataraman S, et al: MicroRNA 128a increases intracellular ROS level by targeting Bmi-1 and inhibits medulloblastoma cancer cell growth by promoting senescence. PLoS One 2010, 5(6):e10748.

46. Andersen $\mathrm{CL}$, Jensen $\mathrm{J}$, Orntoft TF: Normalization of real-time quantitative reverse transcription-PCR data: a model-based variance estimation approach to identify genes suited for normalization, applied to bladder and colon cancer data sets. Cancer Res 2004, 64(15):5245-50.

47. Genovesi LA, et al: Integrated analysis of miRNA and mRNA expression in childhood medulloblastoma compared with neural stem cells. PLoS One 2011, 6(9):e23935.

48. Schuller $U$, et al: Acquisition of granule neuron precursor identity is a critical determinant of progenitor cell competence to form Shh-induced medulloblastoma. Cancer Cell 2008, 14(2):123-34.

49. Yang ZJ, et al: Medulloblastoma can be initiated by deletion of Patched in lineage-restricted progenitors or stem cells. Cancer Cell 2008, 14(2):135-45.

50. Grammel D, et al: Sonic hedgehog-associated medulloblastoma arising from the cochlear nuclei of the brainstem. Acta Neuropathol 2012, 123(4):601-14.

51. Gibson P, et al: Subtypes of medulloblastoma have distinct developmental origins. Nature 2010, 468(7327):1095-9.

52. Meyer SU, Pfaffl MW, Ulbrich SE: Normalization strategies for microRNA profiling experiments: a 'normal' way to a hidden layer of complexity? Biotechnol Lett 2010, 32(12):1777-88.

53. Mestdagh $P$, et al: A novel and universal method for microRNA RT-qPCR data normalization. Genome Biol 2009, 10(6):R64.

54. Chang $\mathrm{KH}$, et al: MicroRNA expression profiling to identify and validate reference genes for relative quantification in colorectal cancer. $B M C$ Cancer 2010, 10:173

55. Shen $Y$, et al: Identification of miR-23a as a novel microRNA normalizer for relative quantification in human uterine cervical tissues. Exp Mol Med 2011, 43(6):358-66.

56. Viprey VF, Corrias MV, Burchill SA: Identification of reference microRNAs and suitability of archived hemopoietic samples for robust microRNA expression profiling. Anal Biochem 2011, 421(2):566-72.
57. Wotschofsky $Z$, et al: Reference genes for the relative quantification of microRNAs in renal cell carcinomas and their metastases. Anal Biochem 2011, 417(2):233-41.

58. Schaefer $A$, et al: Suitable reference genes for relative quantification of miRNA expression in prostate cancer. Exp Mol Med 2010, 42(11):749-58.

59. Chen $\mathrm{L}$, et al: The role of microRNA expression pattern in human intrahepatic cholangiocarcinoma. J Hepatol 2009, 50(2):358-69.

60. Jensen SG, et al: Evaluation of two commercial global miRNA expression profiling platforms for detection of less abundant miRNAs. BMC Genomics 2011, 12:435.

61. Wang B, et al: Systematic evaluation of three microRNA profiling platforms: microarray, beads array, and quantitative real-time PCR array. PLoS One 2011, 6(2):e17167.

62. Pera MF, et al: Isolation, characterization, and differentiation of human embryonic stem cells. Methods Enzymol 2003, 365:429-46.

63. Peh GS, et al: CD133 expression by neural progenitors derived from human embryonic stem cells and its use for their prospective isolation Stem Cells Dev 2009, 18(2):269-82.

64. Bertram CM, et al: Effective adenovirus-mediated gene transfer into neural stem cells derived from human embryonic stem cells. Stem Cells Dev 2010, 19(4):569-78.

65. Ihaka R, Gentleman R: R: A Language for Data Analysis and Graphics. Journal of Computational and Graphical Statistics 1996, 5(3):299-314.

66. Livak KJ, Schmittgen TD: Analysis of relative gene expression data using real-time quantitative PCR and the 2(-Delta Delta C(T)) Method. Methods 2001, 25(4):402-8.

doi:10.1186/1756-0500-5-507

Cite this article as: Genovesi et al:: Identification of suitable endogenous control genes for microRNA expression profiling of childhood medulloblastoma and human neural stem cells. BMC Research Notes 2012 5:507.

\section{Submit your next manuscript to BioMed Central and take full advantage of:}

- Convenient online submission

- Thorough peer review

- No space constraints or color figure charges

- Immediate publication on acceptance

- Inclusion in PubMed, CAS, Scopus and Google Scholar

- Research which is freely available for redistribution 